\title{
The relationship between skipping breakfast and depression among workers with a focus on psychosocial factors
}

\section{Eisho Yoshikawa}

Nippon Medical school

Daisuke Nishi ( $\nabla$ d-nishi@m.u-tokyo.ac.jp )

Yutaka J. Matsuoka

National cancer center Japan

\section{Research note}

Keywords: breakfast, depression, trait aggression, physical exercise

Posted Date: July 10th, 2019

DOI: https://doi.org/10.21203/rs.2.11219/v1

License: (a) (1) This work is licensed under a Creative Commons Attribution 4.0 International License. Read Full License 


\section{Abstract}

Objective Previous studies have indicated that skipping breakfast is not only detrimental to physical health, but is also associated with depression. Myriad psychosocial factors are associated with both skipping breakfast and depression. However, previous research failed to account for these potential psychosocial factors. The aims of this study were to (a) explore the factors associated with breakfast consumption, and (b) investigate the association between the frequency of breakfast consumption and depression, adjusted for various forms of psychosocial confounders by analyzing previously collected data concerning Japanese workers. Result Participants were 575 employee across three Japanese worksites. Depressive symptoms were assessed using the Center for Epidemiologic Studies Depression Scale (CESD). Frequency of breakfast consumption was categorized into 6 levels. Multi-regression analysis suggested that skipping breakfast was associated with high trait aggression, living alone, and low levels of physical exercise. Logistic regression suggested that skipping breakfast was associated with depression, even after adjusting for potential psychosocial factors including trait aggression, resilience, physical exercise, consumption of fish and fried food, social support, and life events cannot be ruled out.

\section{Abstract \\ Objective}

Previous studies have indicated that skipping breakfast is not only detrimental to physical health, but is also associated with depression. Myriad psychosocial factors are associated with both skipping breakfast and depression. However, previous research failed to account for these potential psychosocial factors. The aims of this study were to (a) explore the factors associated with breakfast consumption, and (b) investigate the association between the frequency of breakfast consumption and depression, adjusted for various forms of psychosocial confounders by analyzing previously collected data concerning Japanese workers.

\section{Result}

Participants were 575 employee across three Japanese worksites. Depressive symptoms were assessed using the Center for Epidemiologic Studies Depression Scale (CESD). Frequency of breakfast consumption was categorized into 6 levels. Multi-regression analysis suggested that skipping breakfast was associated with high trait aggression, living alone, and low levels of physical exercise. Logistic regression suggested that skipping breakfast was associated with depression, even after adjusting for potential psychosocial factors including trait aggression, resilience, physical exercise, consumption of fish and fried food, social support, and life events cannot be ruled out.

Key words; breakfast, depression, trait aggression, physical exercise

\section{Introduction}


Depression is one of the most common mental disorders among Japanese workers [1]. Moreover, depression has a significant negative impact on the performance of both the individual worker and the company [2]. Data suggest that skipping breakfast is detrimental to both physical[3] and mental health, including depression. Previous research has also reported an association between skipping breakfast and poor sleep quality [4], which is associated with mental disorders and increased probability of depression[5, 6]. Additionally, breakfast consumption is associated with the regulation of the circadian clock [7], while disruption of circadian rhythms is viewed as a risk factor for depression. Moreover, breakfast consumption lowers cortisol levels [8], and high cortisol levels have been identified as another risk factor for subsequent diagnosis of depression [9]. This convergence of evidence suggests a potential mechanism through which skipping breakfast leads to development of depression. In fact, many epidemiological studies have demonstrated a negative association between breakfast consumption and depression [10-20] although some studies have failed to identify this relationship [21, 22].

On the other hand, many biological [23], psychological[24-26], social[27, 28] , and lifestyle factors[29, 30] are associated with the depression. Similarly, many psychosocial factors are associated with both skipping breakfast and depression. However, many previous attempts to investigate the association between breakfast consumption and depression failed to account for various potential confounding factors. The aims of the current study are to (a) explore the psychosocial factors associated with frequency of breakfast consumption, and (b) analyze previously collected data to investigate the association between breakfast consumption and depression while accounting for a wide range of psychosocial factors [31,32] .

\section{Methods}

This study was approved by the institutional review boards of both the National Disaster Medical Center and the companies involved. All participants have provided written informed consent.

594 employees were selected from across three worksites. Data from 19 participants were excluded due to incomplete responses for the subscales used. Thus, data for 575 participants were included in the analysis performed in this study.

Self-report data were collected on sex, marital status, educational background, and job status. The Center for Epidemiologic Studies Depression (CES-D) questionnaire was administered to assess depression during a one-week period [33] . Participants with a CES-D score $\geq 16$ are considered to meet criteria for a diagnosis of depression [33] . Participants were asked to provide information concerning their frequency of breakfast consumption on the lifestyle questionnaire by responding to the following question: "How often do you eat breakfast?" There were six response options: $<1$ time/month, 1-3 times/month, 1-2 times/week, 3-4 times/week, 5-6 times/week, or daily.

Regarding dietary factors that have been shown to be associated with decreased probability of depression , participants were asked to report their frequency of fish consumption and fried food consumption. Six response options given for each question: almost never, 1-3 times/month, 1-2 times/week, 3-4 times/week, 5-6 times/week, and every day. Frequency of physical exercise was also assessed, and four response options were given for each question: never, 1-3 times a month, 1-2 times a week, and 3 or more 
times per week.Regarding psychosocial factors, the 14-item Resilience Scale (RS-14) was administered to assess resilience [34] , the 24-item Buss-Perry Aggression Questionnaire (BPAQ) to assess trait aggression [35], and the short version of the Social Support Questionnaire (SSQ) to assess social support [36] . Finally, we embedded a one-item questionnaire to assess whether participants had experienced major life events during the past month.

Data were expressed as means (standard deviation) and percentages for both continuous and categorical variables. The association of confounding factors with frequency of breakfast consumption was examined using linear regression analysis for continuous variables and the chi-square test for categorical variables. A strong association was identified between marital status and living alone. We therefore omitted marital status from the subsequent multivariate analysis to avoid distortion of the results due to multicollinearity. Additionally, we conducted multiple regression analysis by entering variables relevant to significant univariate association to explore the associated factor of breakfast consumption.

To examine the association between frequency of breakfast consumption and depression, we performed logistic regression analysis and calculated the odds ratios (ORs) and $95 \%$ confidence intervals (Cls) of depression for frequency of breakfast consumption, with those who consumed breakfast every day used as the reference group. The first model used depression as the dependent variable, and frequency of breakfast consumption as the independent variable. The second model was adjusted for age, sex, marital status, job status, and education level. The third model was further adjusted for frequency of fish consumption, fried food consumption, and physical exercise. Finally, the fourth model was further adjusted for resilience, trait aggression, social support, and life events. All of the analyses were performed using SPSS, version 25 (SPSS Inc., Chicago). Alpha levels were all set at $p<0.05$.

\section{Results}

Results of the univariate analysis indicated that low frequency of breakfast consumption was significantly associated with younger age, being unmarried, living alone, low frequency of fish consumption, low frequency of exercise, low resilience, and high trait aggression (see Table 1). Furthermore, multipleregression analysis indicated that living alone $(\beta=-012, t=-2.58, p=0.01), B P A Q(\beta=-012, t=-2.83, p<$ 0.01 ), and frequency of physical exercise $(\beta=0.12, t=-2.93, p<0.01)$ were associated with frequency of breakfast consumption. The ORs of depressive symptoms according to frequency of breakfast consumption are shown in Table 2. In all models, there was a significant association between frequency of breakfast consumption and depression.

\section{Discussion}

In this study, we found that skipping breakfast was significantly related to depression among Japanese workers, even after controlling for a wide range of factors previously demonstrated to be associated with depression. Results of the present study are consistent with those reported in the literature. Most previous studies did not control for lifestyle, or psychological or dietary factors. However, a cohort study demonstrated that skipping breakfast presented an increased risk of depressive symptoms, even after 
adjusting for other dietary factors that have been suggested to decrease depressive symptoms, such as carbohydrate, folate, magnesium, and zinc intake [20]

Previous research demonstrated an association between psychological factors and health related behavior. Trait aggression has been demonstrated to be associated with unhealthy behaviors such as excessive drinking, smoking, and irregular eating schedules [37], as well as depression [38]. In the current study, trait aggression was associated with frequency of breakfast consumption, independently adjusted for potential confounders. Additionally, frequency of breakfast consumption was associated with depression, independent of trait aggression. These associations might imply a mediating effect of trait aggression on depression through skipping breakfast[39].

\section{Limitations}

This study had several limitations that may have affected its results. Due to the cross-sectional nature of the study, a potential reverse causality, whereby symptoms of depression affect the consumption of breakfast, cannot be ruled out. Also, because most of the participants were highly educated men employed by a large Japanese company providing good job security and a relatively strong balance between work and reward, the results may not be generalizable to employees in other working environments. Finally, information on analyzed variables was self-reported, leading to the potential for nondifferential misclassification.

\section{Abbreviations}

CES-D: The Center for Epidemiologic Studies Depression SSQ: Short version of Social Support Questionnaire RS-14: 14-item Resilience Scale, Cl: confidence interval, BPAQ: Buss-Perry Aggression Questionnaire: S.D., Standard deviation

\section{Declarations}

\section{Ethics approval and consent to participate}

This study was executed in accordance with the Declaration of Helsinki. This study was approved by the institutional review boards of both the National Disaster Medical Center and the companies. The names of review boards of the company were not described here because belonging to private information of the company. All participants have provided written informed consent. All information of participants were kept confidential throughout the process of study, and the name of the participant was replaced by code not to identify.

\section{Consent for publication}

Not applicable 


\section{Availability of data and materials}

The datasets generated during the current study are not publicly available. When this research plan was approved by the institutional review boards of both the National Disaster Medical Center and the companies, these matters was not included and not discussed. Therefore, we did not obtain consent from the participants to share these datasets. Furthermore, we believe that the matters examined in this research involve potentially sensitive topic for companies, who are not willing to disclose their employees' data in such detail. However, the datasets are available from the corresponding author on reasonable request.

\section{Competing interests}

The authors declare that they have no competing interests.

\section{Funding}

This study was supported by grants from the Foundation for Total Health Promotion and CREST, Japan Science and Technology Agency.

\section{Authors' contributions}

The authors alone are responsible for the content and writing of the paper.EY participated in the study to a significant extent and worked on study conception and design; data collection and carriedout the development of this manuscript and data analysis. DN participated in the study to a significant extent and worked on study conception and design; data collection, analysis, interpretation and in drafting and editing the manuscript. YM participated in the study to a significant extent and worked on study conception and design; data collection, in drafting and editing the manuscript. All authors contributed to the intellectual content of the article and read and approved the submitted manuscript.

\section{Acknowledgments}

The authors thank Koichi Iwata and Toshinari Saeki for their cooperation with the research. We also express special thanks to Mss. Akutsu and Kamoshida for data management and to all participants in this study. We also thank Masami Kashimura for his advice on statistical issues.

\section{References}

1. Nakamura-Taira N, Izawa S, Yamada KC. Stress underestimation and mental health literacy of depression in Japanese workers: A cross-sectional study. Psychiatry Res. 2018;262 December 2017:221-228. 
2. Mykletun A, Overland S, Dahl AA, Krokstad S, Bjerkeset O, Glozier N, et al. A population-based cohort study of the effect of common mental disorders on disability pension awards. Am J Psychiatry. 2006;163:1412-8.

3. Smith KJ, Gall SL, McNaughton SA, Blizzard L, Dwyer T, Venn AJ. Skipping breakfast: longitudinal associations with cardiometabolic risk factors in the Childhood Determinants of Adult Health Study. Am J Clin Nutr. 2010;92:1316-25.

4. Katagiri R, Asakura K, Kobayashi S, Suga H, Sasaki S, Three-generation Study of Women on Diets and Health Study Group. Low Intake of Vegetables, High Intake of Confectionary, and Unhealthy Eating Habits are Associated with Poor Sleep Quality among Middle-aged Female Japanese Workers. J Occup Health. 2014;56:359-68.

5. Chang PP, Ford DE, Mead LA, Cooper-Patrick L, Klag MJ. Insomnia in Young Men and Subsequent Depression: The Johns Hopkins Precursors Study. Am J Epidemiol. 1997;146:105-14.

6. Perlis ML, Smith LJ, Lyness JM, Matteson SR, Pigeon WR, Jungquist CR, et al. Insomnia as a Risk Factor for Onset of Depression in the Elderly. Behav Sleep Med. 2006;4:104-13.

7. Tahara Y, Shibata S. Circadian rhythms of liver physiology and disease: experimental and clinical evidence. Nat Rev Gastroenterol Hepatol. 2016;13:217-26.

8. Witbracht M, Keim NL, Forester S, Widaman A, Laugero K. Female breakfast skippers display a disrupted cortisol rhythm and elevated blood pressure. Physiol Behav. 2015;140:215-21.

9. Herbert J. Cortisol and depression: three questions for psychiatry. Psychol Med. 2013;43:449-69.

10. Ahadi Z, Kelishadi R, Qorbani M, Zahedi H, Aram M, Motlagh ME, et al. Association of Breakfast Intake with Psychiatric Distress and Violent Behaviors in Iranian Children and Adolescents: The CASPIAN- IV Study. Indian J Pediatr. 2016;83:922-9.

11. Fulkerson J a, Sherwood NE, Perry CL, Neumark-Sztainer D, Story M. Depressive symptoms and adolescent eating and health behaviors: a multifaceted view in a population-based sample. Prev Med. 2004;38:865-75.

12. Gollub EA, Weddle DO. Improvements in nutritional intake and quality of life among frail homebound older adults receiving home-delivered breakfast and lunch. J Am Diet Assoc. 2004;104:1227-35.

13. Kwak Y, Kim Y. Association between mental health and meal patterns among elderly Koreans. Geriatr Gerontol Int. 2018;18:161-8.

14. Lee G, Han K, Kim H. Risk of mental health problems in adolescents skipping meals: The Korean National Health and Nutrition Examination Survey 2010 to 2012. Nurs Outlook. 2017;65:411-9.

15. Smith AP. Breakfast Cereal Consumption and Subjective Reports of Health by Young Adults. Nutr Neurosci. 2003;6:59-61. 
16. Lee SA, Park E-C, Ju YJ, Lee TH, Han E, Kim TH. Breakfast consumption and depressive mood: A focus on socioeconomic status. Appetite. 2017;114:313-9.

17. Xu Y, Qi J, Yang Y, Wen X. The contribution of lifestyle factors to depressive symptoms: A cross-sectional study in Chinese college students. Psychiatry Res. 2016;245:243-9.

18. Smith AP. Breakfast and mental health. Int J Food Sci Nutr. 1998;49:397-402.

19. Smith a P, Clark R, Gallagher J. Breakfast cereal and caffeinated coffee: effects on working memory, attention, mood, and cardiovascular function. Physiol Behav. 1999;67:9-17.

20. Miki T, Eguchi M, Kuwahara K, Kochi T, Akter S, Kashino I, et al. Breakfast consumption and the risk of depressive symptoms: The Furukawa Nutrition and Health Study. Psychiatry Res. 2019;273:551-8.

21. Huang C, Momma H, Cui Y, Chujo M, Otomo A, Sugiyama S, et al. Independent and combined relationship of habitual unhealthy eating behaviors with depressive symptoms: A prospective study. J Epidemiol. 2017;27:42-7.

22. Kawada T, Kuratomi Y, Kanai T. Lifestyle determinants of depressive feeling and a feeling of unhappiness among workers: A study in Japan. Work. 2009;:255-260.

23. Caspi A, Sugden K, Moffitt TE, Taylor A, Craig IW, Harrington H, et al. Influence of Life Stress on Depression: Moderation by a Polymorphism in the 5-HTT Gene. 2014;386.

24. Heponiemi T, Elovainio M, Kivimäki M, Pulkki L, Puttonen S, Keltikangas-Järvinen L. The longitudinal effects of social support and hostility on depressive tendencies. Soc Sci Med. 2006;63:1374-1382.

25. Siegler IC, Costa PT, Brummett BH, Helms MJ, Barefoot JC, Williams RB, et al. Patterns of change in hostility from college to midlife in the UNC Alumni Heart Study predict high-risk status. Psychosom Med. 2003;65:738-745.

26. Stewart JC, Fitzgerald GJ, Kamarck TW. Hostility now, depression later? Longitudinal associations among emotional risk factors for coronary artery disease. Ann Behav Med Publ Soc Behav Med. 2010;39:258-66.

27. Kendler KS, Thornton LM, Gardner CO. Stressful life events and previous episodes in the etiology of major depression in women: An evaluation of the "kindling” hypothesis. Am J Psychiatry. 2000;157:12431251.

28. Kendler KS, Pres. A Population-Bases Twin Study of Lifetime Major Depression in Men and Women. Arch Gen Psychiatry. 1999;56:39-44.

29. Li F, Liu X, Zhang D. Fish consumption and risk of depression: a meta-analysis. BMJ. 2016;70:299-304.

30. Liu X, Yan Y, Li F, Zhang D. Fruit and vegetable consumption and the risk of depression: A meta-analysis. Nutrition. 2016;32:296-302. 
31. Nishi D, Uehara R, Yoshikawa E, Sato G, Ito M, Matsuoka Y. Culturally sensitive and universal measure of resilience for Japanese populations: Tachikawa Resilience Scale in comparison with Resilience Scale 14item version. Psychiatry Clin Neurosci. 2013;67:174-81.

32. Yoshikawa E, Nishi D, Kashimura M, Matsuoka YJ. Role of resilience for the association between trait hostility and depressive symptoms in Japanese company workers. Curr Psychol. 2019. doi:10.1007/s12144019-0166-y.

33. Radloff LS. The CES-D Scale: A Self-Report Depression Scale for Research in the General Population. Appl Psychol Meas. 1977;1:385-401.

34. Wagnild GM, Young HM. Development and psychometric evaluation of the Resilience Scale. J Nurs Meas. 1993;1:165-78.

35. Ando A, Soga S, Yamasaki K, Shimai S, Shimada H, Utsuki N, et al. Development of the Japanese version of the Buss-Perry Aggression Questionnaire (BAQ)]. Shinrigaku Kenkyu. 1999;70:384-392.

36. Sarason IG, Sarason BR, Shearin EN, Pierce GR. A Brief Measure of Social Support: Practical and Theoretical Implications. Journal of Social and Personal Relationships. 1987;4:497-510.

37. Izawa S, Nomura S. the relationship of hositlity to health related behaviors, obesity, and hypertension in adolescence. Jpn J Health Psychol. 2006;19:11-9.

38. Stewart JC, Janicki-Deverts D, Muldoon MF, Kamarck TW. Depressive symptoms moderate the influence of hostility on serum interleukin-6 and C-reactive protein. Psychosom Med. 2008;70:197-204.

39. Baron RM, Kenny DA. The moderator-mediator variable distinction in social psychological research: conceptual, strategic, and statistical considerations. J Pers Soc Psychol. 1986;51:1173-1182.

\section{Tables}

Due to technical limitations, tables 1 and 2 are only available as downloads in the supplemental files section.

\section{Supplementary Files}

This is a list of supplementary files associated with this preprint. Click to download.

- Table2.jpg

- Table1.jpg 